\title{
Effect of Nonlinear Elasticity on Interband and Intersubband Transition Energies in GaN/AlN Superlattices
}

\author{
S.P. ŁEPKOWSKI \\ Unipress, Institute of High Pressure Physics \\ Polish Academy of Sciences \\ Sokołowska 29, 01-142 Warszawa, Poland
}

\begin{abstract}
We present a theoretical study of the effect of nonlinear elasticity on interband and intersubband transition energies in wurtzite GaN/AlN superlattices. The effect of nonlinear elasticity is considered by taking into account the changes of elastic constants caused by the intrinsic hydrostatic pressure, originating from lattice misfit between constituents of the superlattice. We show that the influence of the effect of the nonlinear elasticity on the interband and intersubband transition energies in the GaN/AlN superlattices depends crucially on the strain state of structures, in particular on the in-plane lattice constant of the buffer or the substrate. For the superlattices strained to AlGaN buffers with small aluminum concentrations, the effect of nonlinear elasticity increases significantly the interband transition energy and decreases the intersubband transition energy. For the superlattices strained to AlGaN buffers with large aluminum concentrations, the effect of nonlinear elasticity leads to a decrease in the interband transition energy and an increase in the intersubband transition energy.
\end{abstract}

PACS numbers: 78.67.Pt, 62.20.Dc, 77.65.Ly, 77.65.-j

\section{Introduction}

Determination of the built-in mechanical and electrical fields plays a crucial role in description of the electronic states in nitride nanostructures. Due to the presence of large internal strains and piezoelectric fields in these structures, the conventional linear theories of elasticity and piezoelectricity have turned out to be insufficient [1-10]. Recently, higher order electromechanical effects, including the nonlinear piezoelectricity [1-3], electromechanical coupling [4-6], and nonlinear elasticity [7-10] have been studied in nitride semiconductors and their heterostructures. 
In this paper, we focus on the effect of the nonlinear elasticity and discuss its influence on the interband and intersubband transition energies in GaN/AlN superlattices (SLs). We show that taking into account the changes of the elastic constants caused by the intrinsic hydrostatic stress, originating from the lattice misfit between constituents of the SLs, leads to significant modifications of the interband and intersubband transition energies in these structures. The effects of nonlinear piezoelectricity and electromechanical coupling are taken into account in calculations but they are not discussed separately.

\section{Strain and electric field in nitride superlattices}

Let us start from the generalized Hook law, which for a wurtzite SL grown in the [0001] direction, takes the following matrix form:

$$
\begin{aligned}
& {\left[\begin{array}{c}
\sigma_{\perp} \\
\sigma_{\perp} \\
0
\end{array}\right]=\left[\begin{array}{lll}
C_{11}\left(P_{\text {int }}\right) & C_{12}\left(P_{\text {int }}\right) & C_{13}\left(P_{\text {int }}\right) \\
C_{12}\left(P_{\text {int }}\right) & C_{11}\left(P_{\text {int }}\right) & C_{13}\left(P_{\text {int }}\right) \\
C_{13}\left(P_{\text {int }}\right) & C_{13}\left(P_{\text {int }}\right) & C_{33}\left(P_{\text {int }}\right)
\end{array}\right]\left[\begin{array}{l}
\varepsilon_{x x} \\
\varepsilon_{y y} \\
\varepsilon_{z z}
\end{array}\right]} \\
& -\left[\begin{array}{l}
e_{31} \\
e_{31} \\
e_{33}
\end{array}\right] E_{z},
\end{aligned}
$$

where $\sigma_{\perp}$ is the biaxial stress due to the lattice misfit between constituents, $\varepsilon_{i i}$ are the diagonal components of the strain tensor, $E_{z}$ is the $z$-component of the electric field, $e_{31}$ and $e_{33}$ are piezoelectric coefficients, and $C_{i j}\left(P_{\text {int }}\right)$ are the pressure-dependent elastic constants. The first term on the right hand side of Eq. (1) takes into account the effect of the nonlinear elasticity since the elastic constants are pressure-dependent. The second term on the right hand side of this equation describes the converse piezoelectric effect and it is responsible for the electromechanical coupling effect [4-6]. The intrinsic hydrostatic pressure $P_{\text {int }}$, originating from the lattice misfit between constituents of a SL, equals $P_{\text {int }}=-\frac{2}{3} \sigma_{\perp}[10]$. From Eq. (1), one gets (i) the equality $\varepsilon_{x x}=\varepsilon_{y y}$ and (ii) the expressions for $\varepsilon_{z z}$ and $\sigma_{\perp}$ in terms of $\varepsilon_{x x}$ and $E_{z}$ :

$$
\begin{aligned}
\varepsilon_{z z} & =-\frac{2 C_{13}\left(P_{\text {int }}\right)}{C_{33}\left(P_{\text {int }}\right)} \varepsilon_{x x}+\frac{e_{33}}{C_{33}\left(P_{\text {int }}\right)} E_{z}, \\
\sigma_{\perp} & =\left\{C_{11}\left(P_{\text {int }}\right)+C_{12}\left(P_{\text {int }}\right)-2 \frac{\left[C_{13}\left(P_{\text {int }}\right)\right]^{2}}{C_{33}\left(P_{\text {int }}\right)}\right\} \varepsilon_{x x} \\
& +\left[e_{33} \frac{C_{13}\left(P_{\text {int }}\right)}{C_{33}\left(P_{\text {int }}\right)}-e_{31}\right] E_{z} .
\end{aligned}
$$

Equations (2) and (3) hold for the quantum wells (QWs) and barriers in the SL. To determine the electric fields in the QWs and barriers, $E_{z, \mathrm{qw}}$ and $E_{z \text {,br }}$, one solves the Poisson equation for the SL, and then after using Eq. (2), yields [10]: 


$$
\begin{aligned}
E_{z, \mathrm{qw}} & =\left\{P_{\mathrm{sp}, z, \mathrm{br}}+2\left[e_{31, \mathrm{br}}-\frac{C_{13, \mathrm{br}}\left(P_{\mathrm{int}, \mathrm{br}}\right)}{C_{33, \mathrm{br}}\left(P_{\mathrm{int}, \mathrm{br}}\right)} e_{33, \mathrm{br}}\right] \varepsilon_{x x, \mathrm{br}}-P_{\mathrm{sp}, z, \mathrm{qw}}\right. \\
& \left.-2\left[e_{31, \mathrm{qw}}-\frac{C_{13, \mathrm{qw}}\left(P_{\mathrm{int}, \mathrm{qw}}\right)}{C_{33, \mathrm{qw}}\left(P_{\mathrm{int}, \mathrm{qw}}\right)} e_{33, \mathrm{qw}}\right] \varepsilon_{x x, \mathrm{qw}}\right\} \\
& /\left\{\left[\lambda_{\mathrm{qw}}+\frac{e_{33, \mathrm{qw}}^{2}}{C_{33, \mathrm{qw}}\left(P_{\mathrm{int}, \mathrm{qw}}\right)}\right]+\frac{L_{\mathrm{qw}}}{L_{\mathrm{br}}}\left[\lambda_{\mathrm{br}}+\frac{e_{33, \mathrm{br}}^{2}}{C_{33, \mathrm{br}}\left(P_{\mathrm{int}, \mathrm{br}}\right)}\right]\right\},
\end{aligned}
$$

where $\lambda_{\mathrm{qw}}$ and $\lambda_{\mathrm{br}}$ are the electric permittivities, $P_{\mathrm{sp}, z, \mathrm{qw}}$ and $P_{\mathrm{sp}, z, \mathrm{br}}$ are the spontaneous polarizations, and $L_{\mathrm{qw}}$ and $L_{\mathrm{br}}$ are the widths of the QWs and barriers, respectively. Formula for $E_{z \text {,br }}$ can be obtained from Eq. (4) by changing indices "qw" and "br".

In this paper, we consider the SLs grown coherently on the substrates or buffer layers whose thickness are much larger than the thickness of the SLs, thus the in-plane strain components are given by $\varepsilon_{x x, \mathrm{qw}}=\left(a_{\mathrm{buff}} / a_{\mathrm{qw}}\right)-1$ and $\varepsilon_{x x, \mathrm{br}}=$ $\left(a_{\mathrm{buff}} / a_{\mathrm{br}}\right)-1$, where $a_{\mathrm{buff}}, a_{\mathrm{qw}}$, and $a_{\mathrm{br}}$ are the unstrained lattice constants of the buffer, QWs, and barriers, respectively.

\section{Results and discussion}

We use the above model of strain and electric field in the [0001] wurtzite SLs to study the influence of the nonlinear elasticity effect on interband and intersubband transition energies, $E_{c-v}$ and $E_{c 2-c 1}$, in GaN/AlN SLs. We consider undoped structures coherently grown on AlGaN buffer layers. We choose $L_{\mathrm{qw}}=2.5 \mathrm{~nm}$ and $L_{\mathrm{br}}=5 \mathrm{~nm}$ since for these SLs, energies $E_{c 2-c 1}$ are close to $0.8 \mathrm{eV}$ and correspond to the optical communication wavelength range. The electronic states in the SLs have been determined by solving the Schrödinger equation with the $\boldsymbol{k} \cdot \boldsymbol{p}$ Hamiltonian and periodic boundary conditions for the envelope wave functions [10]. All material parameters used in the calculations are listed in Ref. [8]. In particular, we have used the biaxial strain dependence of $e_{31}$ and $e_{33}$ to take into account the effect of nonlinear piezoelectricity [1].

In Fig. 1, we show the values of $P_{\mathrm{int}, \mathrm{qw}}$ and $P_{\mathrm{int}, \mathrm{br}}$, as a function of aluminum

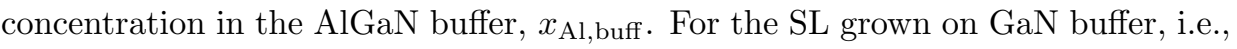
for $x_{\mathrm{Al} \text {,buff }}=0$, the $P_{\mathrm{int} \text {,br }}$ equals almost $-8 \mathrm{GPa}$ (tensile strained barriers) while $P_{\text {int,qw }}$ is close to zero (unstrained QWs). Then, an increase in $x_{\mathrm{Al}, \text { buff }}$ leads to (i) an increase in $P_{\text {int,qw }}$ because compressive strain in the QWs increases, (ii) and an increase in $P_{\text {int,br }}$ due to a decrease in the tensile strain in the barriers. For the SL grown on AlN buffer, i.e., for $x_{\mathrm{Al} \text {,buff }}=1, P_{\mathrm{int}}$,br nearly vanishes while $P_{\mathrm{int}, \mathrm{qw}}$ exceeds 8 GPa.

In Fig. 2 we present the values of $E_{c-v}$ and $E_{c 2-c 1}$, obtained for GaN/AlN SLs using the linear (i.e., stress-independent elastic constants) and nonlinear elastic theories, as a function of $x_{\mathrm{Al} \text {,buff }}$. One can see that the influence of the effect of nonlinear elasticity on $E_{c-v}$ and $E_{c 2-c 1}$ depends crucially on $x_{\mathrm{Al} \text {,buff }}$, i.e., on the 


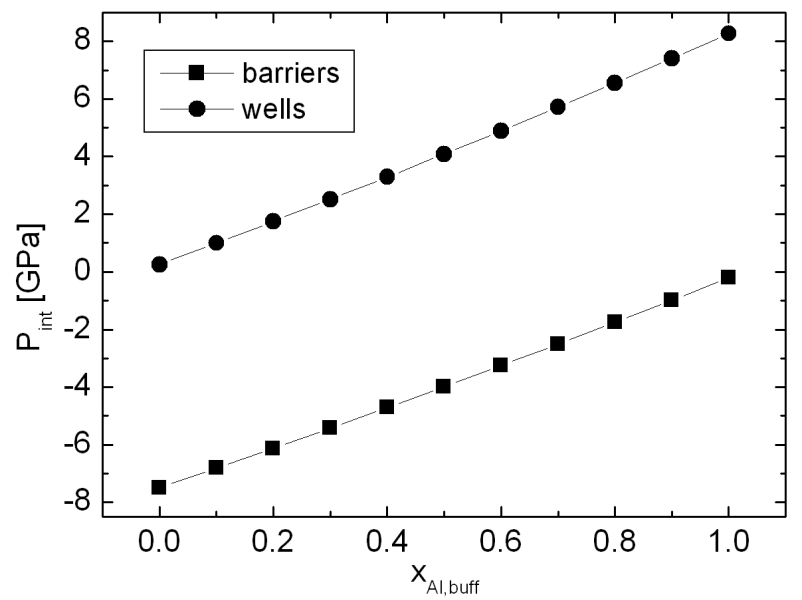

Fig. 1. The values of the intrinsic hydrostatic pressures in the wells (circles) and in the barriers (squares) for the GaN/AlN SLs as a function of aluminum concentration in the AlGaN buffer layer.

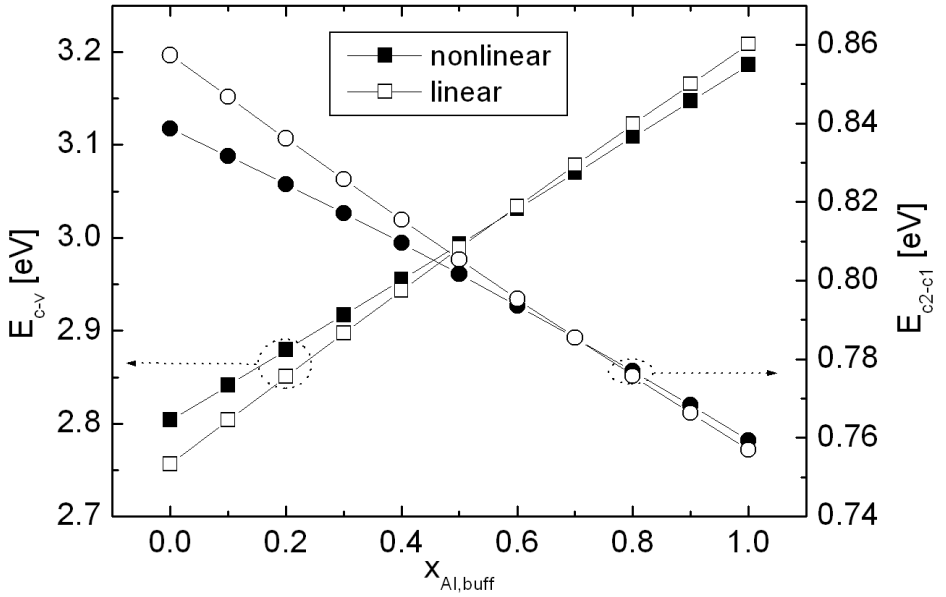

Fig. 2. The energies of the interband (squares) and intersubband (circles) transitions obtained using the nonlinear and linear elastic theories for the GaN/AlN SLs as a function of aluminum concentration in the AlGaN buffer layer.

strain state of the SL. For the SLs strained to AlGaN buffers with small $x_{\mathrm{Al} \text {,buff }}$, the effect of nonlinear elasticity increases significantly $E_{c-v}$ and decreases $E_{c 2-c 1}$.

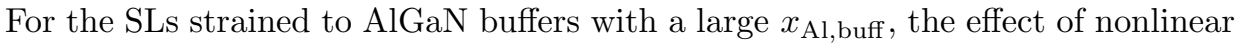
elasticity leads to a decrease in $E_{c-v}$ and an increase in $E_{c 2-c 1}$. 


\section{Conclusions}

In summary, we have studied the influence of the effect of nonlinear elasticity on $E_{c-v}$ and $E_{c 2-c 1}$ in GaN/AlN SLs strained to AlGaN buffers with various aluminum concentrations. Our studies reveal that the effect of nonlinear elasticity

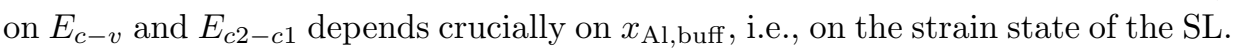
For the SLs strained to AlGaN buffers with a small $x_{\mathrm{Al} \text {,buff }}$, the effect of nonlinear elasticity increases significantly $E_{c-v}$ and decreases $E_{c 2-c 1}$. For the SLs strained

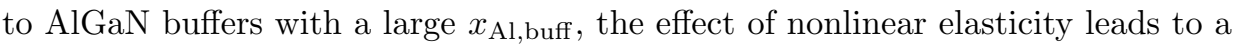
decrease in $E_{c-v}$ and an increase in $E_{c 2-c 1}$.

\section{Acknowledgments}

This work was supported by the STREP project NITWAVE 004170 and by the Polish State Committee for Scientific Research, projects No. 1P03B03729 and N507 011 32/0500. Additionally, the author acknowledges the use of computing facilities at ICM UW.

\section{References}

[1] K. Shimada, T. Sota, K. Suzuki, H. Okumura, Jpn. J. Appl. Phys. 37, L1421 (1998).

[2] G. Vaschenko, D. Patel, C.S. Menoni, N.F. Gardner, J. Sun, W. Gotz, C.N. Tome, B. Clausen, Phys. Rev. B 64, 241308(R) (2001).

[3] F. Bernardini, V. Fiorentini, Phys. Rev. B 64, 085207 (2001).

[4] B. Jogai, J.D. Albrecht, E. Pan, J. Appl. Phys. 94, 6566 (2003).

[5] B. Jogai, J.D. Albrecht, E. Pan, Semicond. Sci. Technol. 19, 733 (2004).

[6] S.P. Łepkowski, J.A. Majewski, Phys. Rev. B 74, 035336 (2006).

[7] S.P. Łepkowski, J.A. Majewski, Solid State Commun. 131, 763 (2004).

[8] S.P. Łepkowski, J.A. Majewski, G. Jurczak, Phys. Rev. B 72, 245201 (2005).

[9] S.P. Łepkowski, J.A. Majewski, Acta Phys. Pol. A 110, 237 (2006).

[10] S.P. Łepkowski, Phys. Rev. B 75, 195303 (2007). 